\title{
Applications of polymer optical fibre grating sensors to condition monitoring of textiles
}

\author{
C.C.Ye ${ }^{1^{*}}$, J.M.Dulieu-Barton ${ }^{2}$, D.J.Webb ${ }^{3}$, C.Zhang ${ }^{3}$, G.-D. Peng ${ }^{4}$, A.R.Chambers ${ }^{2}$, F.J.Lennard ${ }^{1}$, \\ D.D.Eastop ${ }^{1}$ \\ ${ }^{1}$ Textile Conservation Centre, University of Southampton, SO23 8DL, UK \\ 2 School of Engineering Sciences, University of Southampton, SO17 1BJ, UK \\ ${ }^{3}$ Photonics Research Group, Aston University, Birmingham B4 7ET, UK \\ ${ }^{4}$ University of New South Wales, Sydney, Australia
}

\begin{abstract}
Fibre Bragg gratings (FBGs) in polymer optical fibres (POFs) have been used to measure the strain in a woven textile. FBGs in both POFs and silica optical fibres were attached to a woven textile specimen, and their performance characterised. It was demonstrated that the POF FBGs provide improved strain transfer coefficients and reduce local structural reinforcement compared to silica FBGs and therefore make a more suitable proposition for textile monitoring.
\end{abstract}

Keywords: polymer optical fibre, fibre Bragg grating, strain sensor, textile, tapestry

\section{1, INTRODUCTION}

Tapestries are hand-woven textiles that are often large and heavy, so that the strain imposed by their own weight may be the key factor in their deterioration ${ }^{[1,2]}$. Previous experimental investigations using standard silica FBGs have shown that they were an attractive prospect for monitoring the deformations that occur in tapestries ${ }^{[2]}$. However it has been shown ${ }^{[3]}$ that silica optical fibres did not produce an effective strain transfer and that the reinforcement introduced by the sensor attachment was significant ${ }^{[3]}$. The purpose of this paper is to demonstrate that POFs provide an improved performance over silica fibres in applications to textiles. This work also represents what is to the authors' knowledge the first application of POF FBG technology away from the optical bench.

The implementation of FBGs in single mode POFs has advanced dramatically ${ }^{[4]}$. The majority of POFs are based on polymethylmethacrylate (PMMA), which has been shown to be photosensitive. Such POF-based FBGs offer significant advantages over silica-based devices for application to tapestries and textiles, as POFs can withstand and measure much higher strains than those possible with a silica fibre. In particular, the Young's modulus of PMMA POF is approximately 25 times smaller than that of a silica fibre ${ }^{[5]}$ which is more compatible with the modulus of the textile. In this paper, studies on single mode POF-based FBGs attached to a woven wool textile specimen are described. The step-index single-mode POF FBGs were photoinscribed using a $325 \mathrm{~nm} \mathrm{HeCd}$ laser at Aston University in fibre fabricated at UNSW, Sydney. POF and silica-based FBGs were attached to a woven textile specimen with different adhesives and their performance characteristics determined under quasi-static loading. The performance of the FBG/adhesive installations are characterised in terms of strain transfer and reinforcement. To provide an independent measure of strain in a non-contact fashion a digital image correlation (DIC) system $^{[6]}$ was used to obtain full-field data.

\section{2, TEXTILE SPECIMEN AND EXPERIMENTAL DETAILS}

The textile specimen was $49 \mathrm{~mm}$ wide, $0.57 \mathrm{~mm}$ thick and $340 \mathrm{~mm}$ long. Both the warp and weft yarns on the textile are wool with a spacing of $0.8 \mathrm{~mm}$ in the warp direction and $1.7 \mathrm{~mm}$ in the weft direction. Four FBGs were bonded to the central part of the specimen, with the axis of each FBG parallel to the weft yarns. The centres of the four FBGs are respectively on the corners of a rectangle, which is $16 \times 50 \mathrm{~mm}$. The specimen is loaded in the weft direction as this is how tapestries are hung. The Bragg wavelengths of the two POF-based FBGs, POF-FBG-1 and POF-FBG-2, were 1562 and $1565 \mathrm{~nm}$, respectively. For the silica FBGs, Si-FBG-3 and Si-FBG-4, the Bragg wavelengths were 1542 and 1547 $\mathrm{nm}$ respectively. POF-FBG-1 and Si-FBG-3 were bonded to the textile surface with two part epoxy Araldite 2015, whilst POF-FBG-2 and Si-FBG-4 were with the PVA based conservation adhesives Mowilith DMC2. For each FBG, the area covered with adhesive was $3 \mathrm{~mm}$ wide, and up to $50 \mathrm{~mm}$ long. The thickness of adhesive was up to $0.4 \mathrm{~mm}$.

${ }^{*}$ Email of corresponding author: c.ye@soton.ac.uk

20th International Conference on Optical Fibre Sensors, edited by Julian Jones, Brian Culshaw, Wolfgang Ecke, José Miguel López-Higuera, Reinhardt Willsch, Proc. of SPIE Vol. 7503, 75030M (c) 2009 SPIE - CCC code: 0277-786X/09/\$18 - doi: 10.1117/12.833472 
Since the attenuation in the POF at a wavelength of $1560 \mathrm{~nm}$ is high, each POF FBG was written in a short length of $\operatorname{POF}(\sim 5 \mathrm{~cm})$ with a single mode silica fibre connected to one end using an optical adhesive.

This arrangement allowed sufficient definition for the measurement. The mechanical strength of the connection between the POF and the silica fibre is low, as the section of silica fibre near the connection has the coating stripped and is brittle. To accommodate a stable mounting of the sensor to the textile, the fibre connection and the section of silica fibre with coating stripped as well as a short length of silica fibre with coating was bonded to the textile. The penalty for doing this is that the area with adhesive is comparatively long $(\sim 50 \mathrm{~mm})$. Furthermore the maximum strain applied to the specimen is limited by the weak fibre connection. These problems would not exist if the Bragg wavelengths of the POF FBGs were shorter; for example, the attenuation at $800 \mathrm{~nm}$ is approximately 100 times less than at $1550 \mathrm{~nm}^{[7]}$.

The specimen was mounted in an Instron 5569 test machine in wedge grips; the distance between the grips was $240 \mathrm{~mm}$. For quasi static tests, a constant displacement of $10 \mathrm{~mm} /$ minute was applied to the specimen. The deviation of the load was typically $\pm 0.7 \mathrm{~N}$. The Bragg wavelengths of the FBGs were recorded using a Swept Laser Interrogator (FBG-SLI) by Micron Optics. The resolution of measured wavelength is $1 \mathrm{pm}$, corresponding approximately to $1 \mu \varepsilon$. The wavelength range of FBG-SLI is from 1520 to $1570 \mathrm{~nm}$. During the tests, a LaVision DIC system ${ }^{[8]}$ with a single camera was used to monitor the strain over the entire specimen. Data were collected from the side of the specimen without adhesives, to maximise the strain accuracy of DIC by eliminating any possible effects of adhesives on the image correlation processing. Tthe size of the interrogation window was chosen to be $32 \times 32$ pixels, corresponding to $3 \times 3$ $\mathrm{mm}$ in the specimen. For the area away from the FBGs and adhesive, the uncertainty of the measured strain from DIC is reduced by averaging over a number of interogation windows, and is estimated to be $0.06 \%$. For the area with a FBG bonded, the strain from DIC is a 'virtual extensometer' measurement ${ }^{[8]}$ taken by correlating the interrogation cells at either end of the $20 \mathrm{~mm}$ line overlapped the FBG. The uncertainty for the measured strain is estimated to be $0.2 \times 2 / 214$ $=0.19 \%$.

\section{3, QUASI STATIC TESTS}

In the first test a maximum load of $22 \mathrm{~N}$ was applied to the specimen to provide the strain map shown in Figure 3 . The strain values in the two areas covered by Araldite (POF-FBG-1 and Si-FBG-3) are significantly lower than the rest of the sample. The strain in the area where a silica fibre Si-FBG-3 was bonded with DMC2 is also lower than the surrounding areas, while the strain in the area of the POF-FBG-2 bonded with DMC2 is closer to that of the background material.

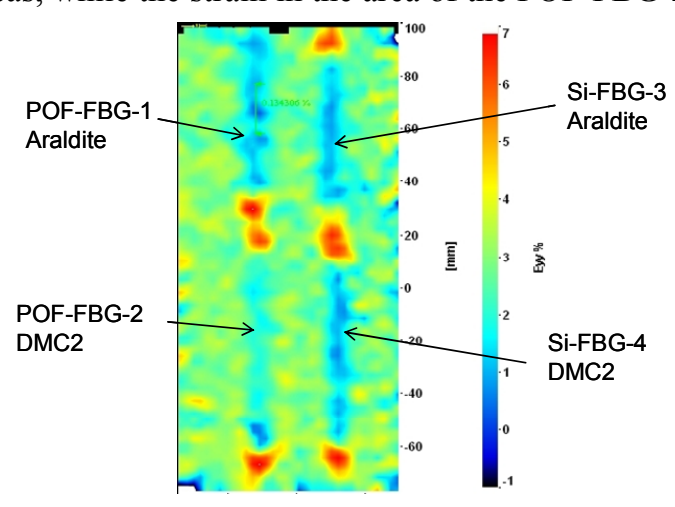

Figure 3 The strain map obtained from DIC. Load applied to the specimen: $20 \mathrm{~N}$

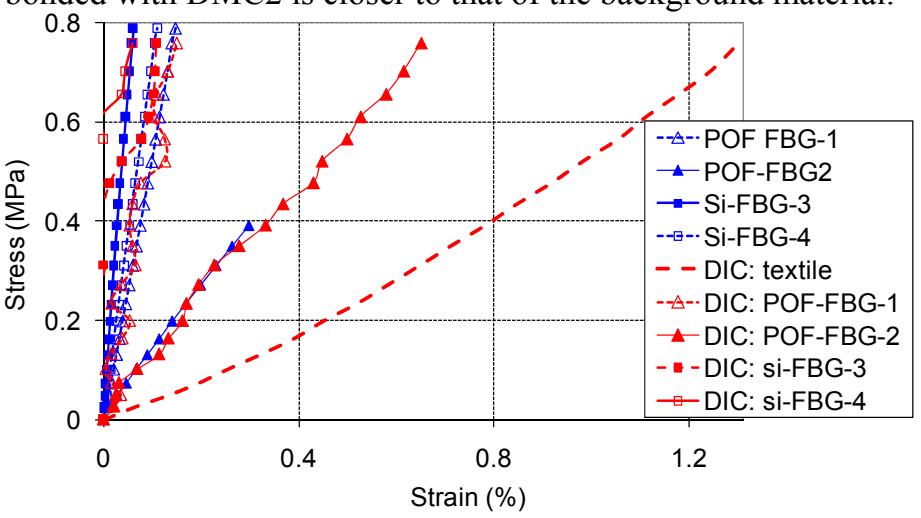

Figure 4 Stress plotted against strains from FBGs and from DIC for different locations.

In Figure 4 the recorded stress is plotted against the strains from the different FBGs and from DIC for different locations. The stress is the total load divided by the area of cross section of the textile specimen. The strains from FBGs were obtained using the measured strain sensitivity of $1.33 \mathrm{pm} / \mu \varepsilon$ for POF FBGs and $1.20 \mathrm{pm} / \mu \varepsilon$ for silica fibre FBGs. For POF-FBG-2, it was not possible to record strain data when the stress was greater than $0.4 \mathrm{MPa}$ since the maximum wavelength of the interrogation system was exceeded. The strain from the POF FBG is greater than that from the silica FBG with the same adhesive. The recorded strain from each type of FBGs with DMC2 is greater than that with Araldite 2015. The strains from the two POF FBGs match the corresponding strains from DIC, although the one bonded using the epoxy adhesive shows much more scatter. The strains from DIC for the areas where the two silica FBGs were bonded are 


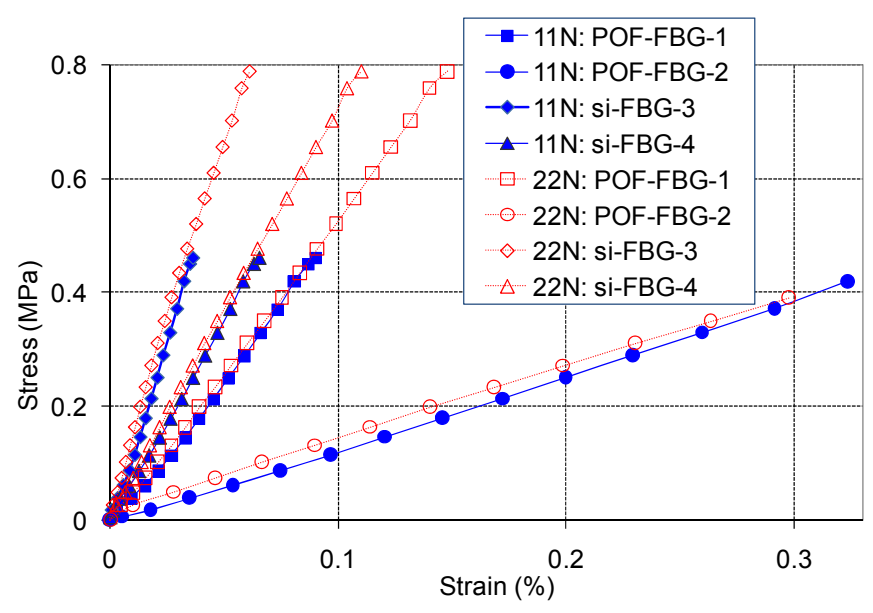

Figure 5 Stress plotted against strains from FBGs for 11 $\mathrm{N}$ quasi-static test (solid blue markers and lines) and 22 $\mathrm{N}$ quasi-static test (open red markers and lines).

noisy, and even became negative when the load was small, indicating the unreliability of the DIC data in this area. This shows that the strain resolution of the DIC system is not enough to measure accurately strains smaller than $0.2 \%{ }^{[3]}$.

The second quasi-static test was done with a maximum load of $11 \mathrm{~N}$. The stress-strain curves for the four gratings obtained from the two quasi-static tests are shown in Figure 5. This demonstrates that the repeatability in both silica and POF sensors are reasonably good. The strain from POF-FBG-2 during $11 \mathrm{~N}$ test was approximately $0.02 \%$ greater than that during $22 \mathrm{~N}$ test for a given stress. This is likely due to the time between the two tests being too short for POF-FBG2 to relax completely, since it was observed that when the load applied to the specimen was removed, the Bragg wavelength of POF-FBG2 relaxed to original value much slower than other FBGs.

\section{4, STRUCTURAL REINFORCEMENT AND STRAIN TRANSFER}

Using the measured data given in Figure 4 it is possible to calculate the strain transfer coefficients and reinforcement coefficients. The strain transfer coefficient is denoted as $R_{I}$ and is the ratio of the strain measured from the FBGs divided by the strain measured by the DIC in the area local to the sensor, i.e. $R_{I}=\varepsilon_{F B G} / \varepsilon_{D I C L}$. The reinforcement coefficient is denoted as $R_{2}$ and is denoted as the ratio of strain measured by the DIC local to the sensor to that away from the sensor $R_{2}=\varepsilon_{D I C L} / \varepsilon_{D I C A}$. The strains $\varepsilon_{F B G}, \varepsilon_{D I C L}$ and $\varepsilon_{D I C A}$ for each sensor/adhesive combination are given in Table 1 along with the two coefficients $R_{1}$ and $R_{2}$. Ideally $\mathrm{R}_{1}$ should be close to unity and for the POFs this is the case. However for the SiFBGs this is not the case. The uncertainty in $\varepsilon_{D I C L}$ local to the silica FBGs is a key consideration ${ }^{[3]}$. In Table 1 a theoretical value for $R_{l}$ also provided using an analytical model given in reference ${ }^{[9]}$. The parameters used in the calculation are as follows: Young's modulus and radius of the sensors $72 \mathrm{GPa} 0.063 \mathrm{~mm}$ for the Si-FBGs and $2.8 \mathrm{GPa}$ $0.093 \mathrm{~mm}$ for the POFs, Young's modulus of the adhesive 1.5 GPa for the epoxy and $0.006 \mathrm{GPa}$ for the PVA. Poisson's ration is taken to be 0.4 for both adhesives. The bonded length of fibre (2L) is $40 \mathrm{~mm}$ for Si-FBG-3 and $50 \mathrm{~mm}$ for the other FBGs. It can be seen from Table 1 that the theory is very close to the actual values for the POFs but there is a large discrepancy in the Si-FBG indicating that errors in the DIC are the cause. It is interesting that the Si-FBG with the DMC2 gives a very low theoretical strain transfer coefficient, indicating that the POFs have a much better compatibility with the DMC2 PVA.

Table 1 Measured strain transfer and reinforcement ratio coefficients

\begin{tabular}{|c|c|c|c|c|c|c|c|c|}
\hline FBG & Adhesive & $\begin{array}{c}\text { Stress } \\
(\mathrm{MPa})\end{array}$ & $\begin{array}{c}\varepsilon_{\text {DICL }} \\
(\mathbf{\%})\end{array}$ & $\begin{array}{c}\varepsilon_{\text {DICA }} \\
(\%)\end{array}$ & $\begin{array}{c}\varepsilon_{\mathrm{FBG}} \\
(\%)\end{array}$ & $\mathrm{R}_{1}$ & $\mathrm{R}_{1}$ (theory) & $\mathrm{R}_{2}$ \\
\hline POF-FBG-1 & Araldite & 0.79 & 0.151 & 1.303 & 0.140 & 0.93 & 0.99 & 0.12 \\
\hline POF-FBG-2 & DMC2 & 0.39 & 0.332 & 0.783 & 0.298 & 0.90 & 0.85 & 0.42 \\
\hline Si-FBG-3 & Araldite & 0.79 & 0.108 & 1.303 & 0.058 & 0.53 & 0.97 & 0.08 \\
\hline Si-FBG-4 & DMC2 & 0.79 & 0.06 & 1.303 & 0.104 & 1.7 & 0.66 & 0.05 \\
\hline
\end{tabular}


In these tests the DIC measured strains for the areas with silica FBGs bonded are too small to be measured by the current DIC system. However the textile specimens have been tested with a silica FBG at a higher strain level ${ }^{[3]}$ and $\mathrm{R}_{1}$ was obtained as $\sim 23 \%$ and $\sim 36 \%$ for DMC2 and Araldite, respectively, still not matching the theoretical values. The amount of reinforcement induced by bonding a fibre sensor can be quantified in terms of the ratio of strains $\mathrm{R}_{2}$; when there is no reinforcement, $R_{2}=1$. The difference in reinforcement ratio $R_{2}$ for the different adhesive/FBG combinations given in Table 1 can be explained in terms of the overall product of Young's modulus and cross section area of the bonded area. The overall product of $E \cdot A$ is the sum of the products of the cured adhesive, optical fibre and the textile covered with adhesive, and can be written as follows:

$$
E \cdot A=E_{a d}\left(w \cdot t-\pi \cdot r_{f}^{2}\right)+E_{f} \cdot \pi \cdot r_{f}^{2}+E_{t} \cdot w \cdot t_{t}
$$

where $E_{a d}, E_{f}$ and $E_{t}$ are Young's moduli for the adhesive, optical fibre and textile, respectively, $r_{f}$ the radius of the optical fibre, $w$ and $t$ the width and length of cured adhesive, and $t_{t}$ the thickness of the textile. The measured Young's modulus and thickness of the textile are $E_{t}=60 \mathrm{MPa}$ and $t_{t}=0.57 \mathrm{~mm}$. The measured $E_{a d}$ values and the $E \cdot A$ values for the two POF fibres, as well as three specimens tested previously ${ }^{[3]}$ are given in Table 2 . The $E \cdot A$ value for the specimen with Araldite is approximately 3 times greater than those with conservation adhesives, which is consistent with the difference in ratio $\mathrm{R}_{2}$. For the two POF FBGs, the $E \cdot A$ value for the specimen with Araldite is more than 10 times greater than those with conservation adhesives. This provides an adequate explanation for the reinforcement.

Table $2 E \cdot A$ values for different specimens

\begin{tabular}{|l|c|c|c|c|}
\hline \multicolumn{1}{|c|}{ Specimens } & $E_{a d}(\mathrm{GPA})$ & $\mathrm{W}(\mathrm{mm})$ & $\mathrm{t}(\mathrm{mm})$ & $\mathrm{E} \cdot \mathrm{A}\left(\mathrm{GPa} \cdot \mathrm{mm}^{2}\right)$ \\
\hline Si-FBG-Araldite & 1.5 & 3.2 & 0.4 & 2.92 \\
\hline Si-FBG-DMC2 & 0.006 & 3.1 & 0.3 & 1.02 \\
\hline Si-FBG-Vinamul & 0.002 & 3.2 & 0.3 & 1.02 \\
\hline POF-FBG-1 (Araldite) & 1.5 & 3 & 0.40 & 1.94 \\
\hline POF-FBG-2 (DMC2) & 0.006 & 3 & 0.22 & 0.18 \\
\hline
\end{tabular}

\section{CONCLUSIONS}

Both POF-based and silica-based FBGs have been used to measured strain in a woven textile specimen. It has been demonstrated that the POFs provide a favourable strain transfer coefficient and that the POFs bonded with PVA offer less structural reinforcement. Further investigation on the feasibility of POF FBGs for tapestry/textile applications is required, including the creep behaviours of the POF FBGs and the effects of temperature and humidity.

\section{ACKNOWLEDGEMENTS}

The support of the EU (FP7 Project PHOSFOS), the UK Engineering and Physical Sciences Research Council and the UK Arts and Humanities Research Council is gratefully acknowledged.

\section{REFERENCES}

1 Dulieu-Barton J.M., Sahin M., Lennard F.J., Eastop D.E. and Chambers A.R. "Assessing the feasibility of monitoring the condition of historic tapestries using engineering techniques”, Key Engineering Materials, 347, pp187-192, 2007

2 Dulieu-Barton J.M., Ye C.C., Chambers A.R., Lennard F.J. and Eastop, D.E., "Optical fibre sensors for monitoring damage in historic tapestries", SEM XI International Congress on Experimental and Applied Mechanics, 2008, Orlando, Florida

3 Ye C.C., Dulieu-Barton J.M., Chambers A.R., Lennard F.J., Eastop D. D., "Condition monitoring of textiles using optical techniques" to be presented in DAMAS'2009 (Beijing, China, 2009)

4 Webb D.J., Kalli K., Carroll, K., Zhang, C., Komodromos M., Argyros A., Large M., Emiliyanov G., Bang O., Kjaer E., "Singlemode polymer optical fiber sensors for high-strain applications", Proceedings of SPIE, Advanced Sensor Systems and Applications III, 6830 art. no. 700467, 2007

5 Silva-Lopez M., Fender A., MacPherson W. N., Barton J. S., Jones J. D. C., Zhao, D., Dobb H., Webb D. J., Zhang L., Bennion, I.,. "Strain and temperature sensitivity of a single-mode polymer optical fiber", Optics Letters 30 (23), pp:3129-3131, 2005

6 Chu T.C., Rason W.F., Sutton M.A. and Peters W.H., "Applications of digital image correlation techniques to experimental mechanics", Experimental Mechanics, 25, 232-244, 1985

7 http://www.paradigmoptics.com/pof/poffaqs.html [Accessed 2009/4/2]

8 LaVision DIC Product-Manual, StrainMaster 3D - Getting Started

9 Li D., Li H., Ren L., Song G. "Strain transferring analysis of fiber Bragg grating sensors", Optical Engineering, 45(2) 024402-18, 2006 